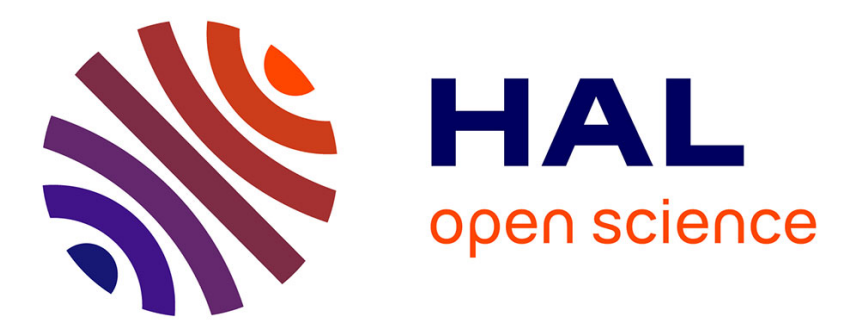

\title{
Jean de Dammartin ou Alexandre de Berneval : qui fut l'inventeur du dessin de la rose occidentale de la cathédrale de Tours?
}

Markus Schlicht

\section{- To cite this version:}

Markus Schlicht. Jean de Dammartin ou Alexandre de Berneval: qui fut l'inventeur du dessin de la rose occidentale de la cathédrale de Tours?. Marcello Angheben, Pierre Martin et Eric Sparhubert (éds.), Regards croisés sur le monument médiéval. Mélanges offerts à Claude Andrault-Schmitt (coll. Culture et société médiévales, 33), Turnhout: Brepols Publishers N.V.,, p. 201-219 et pl. 20, 2018. halshs-01963850

\author{
HAL Id: halshs-01963850 \\ https://shs.hal.science/halshs-01963850
}

Submitted on 21 Dec 2018

HAL is a multi-disciplinary open access archive for the deposit and dissemination of scientific research documents, whether they are published or not. The documents may come from teaching and research institutions in France or abroad, or from public or private research centers.
L'archive ouverte pluridisciplinaire HAL, est destinée au dépôt et à la diffusion de documents scientifiques de niveau recherche, publiés ou non, émanant des établissements d'enseignement et de recherche français ou étrangers, des laboratoires publics ou privés. 


\section{Jean de Dammartin ou Alexandre de Berneval : qui fut l'inventeur du dessin de la rose occidentale de la cathédrale de Tours ?* Markus Schlicht}

La cathédrale de Tours et l'abbatiale Saint-Ouen de Rouen sont pourvues de roses dont les dessins de remplage sont presque identiques : il s'agit, pour la cathédrale tourangelle, de la grande baie occidentale à rose intégrée et, pour l'abbatiale bénédictine rouennaise, de la rose du bras sud du transept (fig. 1 et 2). Les deux réseaux montrent en effet six fois le même motif: partant de l'oculus central ${ }^{1}$, deux grands pétales se déploient et rejoignent le cadre extérieur de la baie, qui adopte la forme d'un cercle à Rouen et celle d'un losange curviligne à Tours. Ces grands pétales s'écartent d'abord l'un de l'autre pour ménager un espace en forme d'amande, garni d'un petit pétale, avant de converger et de se rejoindre près de la périphérie de la rose. Entre ces paires de grands pétales courbes s'intercale un pétale droit plus fin. La disposition des pétales autorise aussi une deuxième lecture : autour de l'oculus central rayonnent six gerbes de trois pétales, le pétale central droit de chaque gerbe étant flanqué de deux pétales courbes divergents ; les six gerbes sont séparées les unes des autres par des petits pétales droits en forme d'amande.

La pointe de tous les pétales, petits et grands, est garnie d'un polylobe à quatre lobes : ceux des petits pétales et ceux des grands pétales incurvés montrent de "vrais » quadrilobes à lobes circulaires, tandis que ceux des grands pétales droits intermédiaires présentent des lobes effilés en pointe (que j'appellerai par la suite des quatre-feuilles). La partie inférieure des pétales est occupée par des arcs brisés redentés qui forment des mouchettes, ou plus précisément des rayons courbes. On trouve deux rayons droits dans les six petits pétales intérieurs, alors que les grands pétales comportent chacun trois rayons courbes: l'un disposé près de l'oculus central, les deux autres, jumelés, plus proches de la périphérie. Les petits écoinçons entre les pétales et le cadre périphérique des roses sont garnis de trèfles (cadrés à Saint-Ouen, libres à Tours). Les différences se cantonnent pour l'essentiel aux adaptations de ce dessin à la forme du cadre, différente à Rouen (rond) et à Tours (losange curviligne).

Cette similitude frappante entre les deux roses, qui semble avoir échappé jusqu'à présent à l'attention des érudits ${ }^{2}$, suppose l'existence d'un lien direct entre Tours et

\footnotetext{
${ }^{1}$ Depuis la récente réfection de la rose de Tours, son oculus central présente un surprenant polylobe à douze lobes.

${ }^{3}$ Parmi les comparaisons formelles indiquées pour la rose de Tours figurent en particulier le "Grand housteau" de la cathédrale de Bourges (Thomas RAPIN et Julien NOBLET, «L'évolution de la façade de la cathédrale de Tours (XII ${ }^{\mathrm{e}}-\mathrm{XVI}{ }^{\mathrm{e}}$ siècles) », Bulletin de la Société archéologique de Touraine, 2001, p. 71) et la Belle cheminée du palais ducal de Poitiers (Thomas RAPIN, « La cathédrale de Tours. La façade. Les campagnes du XV siècle et le programme iconographique du portail central», Congrès archéologique de France, $155^{e}$ session, 1997 Touraine, Paris, Société française d'archéologie, 2003, p. 301-315, en part. p. 313, note 41 ; Thomas RAPIN, « Les Dampmartin, une dynastie de maîtres d'œuvre à la lecture des sources (1365-1469) », Revue historique du
} 
Rouen en ce deuxième quart du XVe siècle, époque à laquelle ont été réalisées ces deux œuvres. Je tenterai donc, dans les lignes qui suivent, de préciser les modalités de ce transfert artistique, en m'interrogeant notamment sur les questions suivantes : Y a-t-il un ou deux auteurs, et quelle est son/leur identité ? De quand datent ces deux roses, et quelle est leur chronologie relative ? Enfin : qui est l'inventeur de ce dessin de remplage ? Faute de sources écrites pertinentes pour trancher cette dernière question, l'argumentation s'appuiera avant tout sur des considérations d'ordre stylistique.

\section{Les auteurs des deux roses}

Il ne saurait être question d'étudier ici l'histoire de la construction de la façade occidentale de la cathédrale de Tours, vaste tâche qui dépasserait largement le cadre que la présente contribution s'est fixé. Rappelons simplement que le frontispice actuel est le résultat d'un admirable rhabillage de l'ancienne façade romane, dont subsistent notamment les tours occidentales. Ce rhabillage fut réalisé tout au long du XVe siècle, les derniers niveaux des tours n'étant achevés qu'en 1507 (tour nord) et 1547 (tour sud) ${ }^{3}$. Le début de ces travaux semble se situer vers 1426: après la destruction du clocher (nord) par la foudre en 1425, le chapitre cathédral se propose l'année suivante, lors d'une fête solennelle commémorant la catastrophe, de réédifier les tours en pierre ${ }^{4}$. La chronologie des travaux est encore assez mal connue et semble complexe: contrairement à ce que l'on attendrait, les premiers travaux n'affectèrent apparemment pas les portails, mais les parties situées juste au-dessus ${ }^{5}$.

À en juger d'après leurs caractères stylistiques, les remplages plaqués ornant la partie de la façade qui surmonte les portails, y compris la grande baie à rose intégrée, peuvent être attribuées au deuxième quart du $\mathrm{XV}^{\mathrm{e}}$ siècle. Le niveau inférieur de ces placages, situé juste au-dessus des gâbles des portails latéraux, forme une sorte de faux triforium. $\mathrm{Au}$ sud comme au nord, deux paires d'arcades majeures regroupent chacune deux lancettes et un tympan composé de deux mouchettes enveloppant un quadrilobe central (fig. 3, en bas). Le quadrilobe, redressé, présente un lobe inférieur s'étirant en pointe ; les mouchettes, têtes orientées vers le bas, le contournent et convergent vers cette pointe. De manière frappante, tous les écoinçons des remplages de la tour nord - aussi bien ceux du tympan, situés entre la tête des lancettes et les mouchettes, que ceux à l'extérieur des arcades majeures - sont garnis de trèfles et de quatre-feuilles libres et asymétriques, dont les lobes s'étirent et se déforment à volonté afin d'épouser parfaitement ces espaces résiduels. Le réseau de ce «faux triforium » de la façade est donc presque identique à celui du « vrai » triforium de la troisième travée, côté sud, de la

Centre-Ouest, IV, $2^{\mathrm{e}}$ semestre 2005, p. 247-271, en part. p. 270 ; Claude ANDRAULT-SCHMITT, La cathédrale de Tours, La Crèche, Geste éditions, 2010, p. 244).

${ }^{3}$ C. Andrault (op. cit. n. 2), p. 90.

4 T. RAPIN, «Les Dampmartin... » (art. cit. n. 2), p. 270 ; l'événement nous est connu grâce à l'ouvrage du chanoine Jean Maan, Sancta et metropolitana ecclesia Turonnensis, Tours, 1667, p. 163, XIV; cf. aussi C. ANDRAULT (op. cit. n. 2), p. 86.

${ }^{5}$ T. RAPIN, « Les Dampmartin... » (art. cit. n. 2), p. 269-271 ; C. ANDRAUlT (op. cit. n. 2), p. 224-225. 
nef (en comptant depuis la croisée), que Claude Andrault date des alentours de 14296. D’ailleurs, ces trèfles et quatre-feuilles fortement déformés occupant les écoinçons se retrouvent aussi dans d'autres édifices de la période, tels que la Grande chapelle de Saint-Seurin de Bordeaux (baie occidentale, entre 1427 et 1444$)^{7}$ ou encore les remplages plaqués du bras nord du transept de la cathédrale Saint-André de la même ville (années 1430 ?).

Compte tenu de la date de leur réalisation vers ou peu après 1429 , il convient donc d'attribuer le « faux triforium » ainsi que les baies plaquées à roses intégrées au-dessus, nécessairement (de peu) postérieurs, au maître d'œuvre Jean de Dammartin. Attesté pour la première fois en 1431 à Tours, Jean a pu prendre la direction du chantier quelques années auparavant. Alors qu'il avait dirigé l'œuvre de la cathédrale du Mans depuis 1421, Jean de Dammartin disparaît en effet des sources relatives à cet édifice en $1425^{8}$. Ne pourrait-on dès lors pas penser que l'architecte a quitté le chantier de SaintJulien du Mans en 1425/1426 pour assumer la maîtrise d'œuvre de Saint-Gatien de Tours dont le chapitre avait pris la décision solennelle, précisément en 1426, de reconstruire les tours en pierre ${ }^{9}$ ? Quoi qu'il en fût, Jean de Dammartin quitta à son tour la maîtrise d'œuvre de Saint-Gatien en 1453 au plus tard, au moment où sa maison située près de la cathédrale fut mise en vente et que son collaborateur Jean Papin lui succéda ${ }^{10}$.

Revenons à présent à la grande baie occidentale de la façade de la cathédrale tourangelle. Compte tenu de la période chronologique (1426/31-1453), de la longue durée pendant laquelle Jean de Dammartin dirigea le chantier et des caractères stylistiques des remplages - comparables à plusieurs créations de son oncle Guy de Dammartin -, il faut sans doute voir en lui le constructeur de la baie maîtresse du frontispice tourangeau ${ }^{11}$.

Quant à la rose du bras sud du transept de Saint-Ouen de Rouen, elle n'a pas été réalisée par le même architecte. En effet, selon toute évidence, il convient de l'attribuer à Alexandre de Berneval, maître d'œuvre de l'abbatiale rouennaise entre 1422 environ et 1441, date de sa mort.

\footnotetext{
${ }^{6}$ En 1429, le duc Jean V de Bretagne fait des dons à l'œuvre de la cathédrale en vue d'achever la nef, dont seules les deux travées orientales avaient alors été construites, cf. C. ANDRAULT (op. cit. n. 2), p. 86 et p. 87, légende de la fig. 59. Cf. aussi T. RAPIN et J. NOBLET (art. cit. n. 2), p. 70, note 20.

7 Pour cette chapelle, cf. Markus SCHLICHT, «La Grande chapelle dite Notre-Dame-de-la-Rose », Autour de Saint-Seurin de Bordeaux : lieu, mémoire et pouvoir des premiers temps chrétiens à la fin du Moyen Âge, (actes du colloque de Bordeaux, 12-14 octobre 2006), dir. I. CARTRON et al., Bordeaux, Éditions Ausonius, 2009 , p. 311-329.

${ }^{8}$ T. RAPIN, « Les Dampmartin... » (art. cit. n. 2), p. 268 et p. 270.

${ }^{9}$ Le lien entre le départ du Mans de Jean de Dammartin et le démarrage du chantier de la façade de Saint-Gatien a déjà été établi par T. RAPIN et J. NOBLET (art. cit. n. 2), p. 71.

${ }^{10}$ T. RAPIN et J. NOBlet (art. cit. n. 2), p. 71 ; T. RAPIN, « Les Dampmartin... » (art. cit. n. 2), p. 271.

11 Cette attribution a déjà été proposée par T. RAPIN, «Les Dampmartin...» (art. cit. n. 2), p. 270 et C. ANDRAUlT (op. cit. n. 2), p. 224-225.
} 
Cette attribution se justifie pleinement par la représentation figurée gravée sur sa dalle funéraire - toujours conservée à Saint-Ouen ${ }^{12}$ - qui montre le maître d'œuvre en pied sous un baldaquin architecturé, tenant de la main droite le petit compas et de l'autre une planche à tracer sur laquelle figure justement le dessin du réseau de la rose du bras sud du transept (fig. 4). L'inscription qui court le long du cadre de la dalle ne laisse aucun doute sur l'identité du défunt, en l'occurrence "Alixandre de Berneval » ${ }^{13}$. Il faut aussi insister sur la précision de ce dessin et sa conformité avec la rose du bras sud : chacun des détails de la rose réelle est scrupuleusement reproduit sur la dalle funéraire. Il en va ainsi du nombre et de l'inclinaison des mouchettes qui subdivisent les pétales, de la distinction entre quadrilobes à lobes arrondis (lorsqu'il s'agit d'un pétale incurvé) et à lobes effilés en pointe (lorsqu'il s'agit d'un pétale droit), ou encore des trèfles meublant les écoinçons entre les pétales et le cercle périphérique de la rose : seuls en reçoivent les écoinçons situés de part et d'autre d'un quatre-feuilles à lobes effilés, tandis que ceux qui s'intercalent entre deux pétales courbes restent dépourvus de redents. L'unique véritable divergence entre les deux œuvres réside dans l'orientation du dessin : celui de la dalle est tourné de $30^{\circ}$ par rapport à la rose construite du bras sud.

\section{La datation des deux roses}

Comme nous l'avons vu ci-dessus, la baie occidentale de Tours n'est pas datée par des documents écrits, les dates de la maîtrise d'œuvre de Jean de Dammartin - pour autant qu'il soit effectivement l'auteur de la rose - ne fournissant qu'une fourchette approximative, en l'occurrence la période comprise entre 1426/31 et 1453 .

A Rouen, la situation se présente de manière bien différente. La rose du bras sud paraît bien datée - trop bien même, comme nous le verrons. En effet, Jean-François Pommeraye affirme dans son ouvrage sur Saint-Ouen, publié en 1662, que les deux roses du transept de Saint-Ouen ont été réalisées en 143914. En dépit de la très grande érudition et du travail scrupuleux dont le savant bénédictin fait preuve par ailleurs ${ }^{15}$, la crédibilité de cette affirmation est entachée par l'anecdote qu'il rapporte à propos de cette rose. Selon Pommeraye, les deux roses du transept de Saint-Ouen auraient été réalisées respectivement par Alexandre de Berneval et son apprenti. La rose réalisée par

\footnotetext{
${ }^{12}$ La dalle se trouve dans la chapelle Sainte-Cécile, dans la partie septentrionale du chevet, redressée contre le mur.

${ }^{13}$ Le texte complet, rédigé en français, de la dalle d'Alexandre de Berneval est le suivant: «Ci gist maistre Alixandre de Berneval, maistre des œuvres de machonnerie du roy nostre sire ou bailliage de Rouen et de ceste église, qui trespassa l'an de grace mil CCCCXL [1441 n.s.], le Ve jour de janvier. Priez Dieu pour l'âme de luy » (transcription d'après André MASSON, L'église abbatiale Saint-Ouen de Rouen (coll. Petites monographies des grands édifices de la France), Paris, H. Laurens, 1927, p. 68; le texte est également reproduit dans Jules QuicherAT, "Documents inédits sur la construction de S. Ouen de Rouen », Bibliothèque de l'école des chartes, 13, 1852, p. 464-476, en part. p. 465 ; il figure déjà dans Jean-François POMMERAYE, Histoire de l'abbaye royale de S. Ouen de Rouen, Rouen, Richard Lallemant, 1662, p. 197).

${ }^{14}$ J.-F. POMMERAYE (op. cit. n. 13), p. 196-197.

${ }^{15}$ Jean-François Pommeraye, bénédictin de la congrégation de Saint-Maur (1617-1697), est notamment l'auteur d'une volumineuse monographie sur la cathédrale de Rouen, parue à Rouen en 1667. Le savant bénédictin rédigea également des études monographiques sur plusieurs abbayes de Rouen, dont celle sur Saint-Ouen est la plus détaillée.
} 
ce dernier ayant été considérée par tous comme étant meilleure et plus belle, Alexandre l'aurait tué par jalousie. Alexandre aurait alors été condamné et exécuté pour ce crime, avant d'être enterré dans l'abbatiale.

Cette légende paraît pour le moins invraisemblable ${ }^{16}$. La rose sud de Saint-Ouen, dont Alexandre fut indubitablement l'auteur (comme l'atteste sa dalle funéraire), présente des formes nettement plus récentes et «modernes » que son pendant septentrional, et ne peut donc pas lui être exactement contemporaine. Même en admettant le contraire, c'est-à-dire une réalisation strictement simultanée des deux roses, celle du nord n'a manifestement pas été considérée comme la plus belle. L'estime suscitée par le dessin de remplages figurant sur la dalle d'Alexandre de Berneval se mesure en effet à sa transposition à l'échelle monumentale non seulement à Rouen et à Tours, mais aussi à Saint-Jacques de Dieppe (fig. 5). La rose ornant la façade occidentale de la paroissiale normande apparaît comme une copie légèrement simplifiée de sa grande sœur rouennaise ${ }^{17}$. Si ce dessin a donc été mis en œuvre à trois reprises au moins, celui de la rose nord de Saint-Ouen ne connut en revanche pas d'imitations ${ }^{18}$.

Enfin, on ne voit pas comment les moines de Saint-Ouen - aussi satisfaits qu'ils eussent été des services d'Alexandre - auraient pu prendre l'initiative d'enterrer à l'intérieur de leur église un meurtrier qu'ils auraient de surcroît honoré par une dalle funéraire somptueuse. Bien entendu, ce type de pierre tombale constitue un honneur insigne dont nous ne connaissons que très peu d'exemples médiévaux en France. Rares sont en effet les architectes qui, à l'instar d'Alexandre de Berneval, reçurent la permission de se faire inhumer dans l'édifice religieux qu'ils avaient érigé, et dont l'effigie en pied, gravée sur la pierre tombale, assurait la mémoire pour les générations futures. Outre le premier maître d'œuvre de Saint-Ouen, resté anonyme à cause de l'effacement de l'inscription de sa dalle, nous ne conservons guère que les célèbres monuments funéraires de Pierre de Montreuil ${ }^{19}$ et d'Hugues Libergier ${ }^{20}$.

\footnotetext{
${ }^{16}$ Elle avait déjà été rejetée par J. QUICHERAT (art. cit. n. 12), p. 476, de même que par A. MASSON (op. cit. n. 12), mais on la trouve encore parfois dans certains textes de vulgarisation.

${ }^{17}$ La datation haute de la rose dieppoise (« avant $1340 »$ ), avancée par Robert de Lasteyrie en 1926, puis reprise par Peter Kurmann en 1983, ne peut à mon sens pas être maintenue. Cf. Peter KuRMANN, "Spätgotische Tendenzen in der europäischen Architektur um $1300 »$, dans Hermann FiLliTZ et Martina PIPPAL (éd), Europäische Kunst um 1300. Akten des XXV. Internationalen Kongresses für Kunstgeschichte, 4.-10. September 1983, Vienne, Cologne, Graz, H. Böhlau, 1986, vol. 6, p. 11-18, en part. p. 13. S'il est vrai que la façade occidentale de Saint-Jacques s'inspire des façades du transept de la cathédrale de Rouen (1281-1330 environ), les remplages montrent partout exclusivement des formes appartenant au répertoire rayonnant; la rose, pour ainsi dire identique à celle de Saint-Ouen, est le seul élément à montrer des formes proto-flamboyantes. Les remplages de cette rose, que l'on pouvait enlever et remplacer sans compromettre la stabilité des autres parties de la façade, ont donc dû être refaits au $\mathrm{XV}^{\mathrm{e}}$ siècle.

${ }^{18} \mathrm{~L}$ 'exemple de comparaison le plus proche, à savoir la rose du bras nord du transept de la cathédrale d'Amiens, ne peut lui être rapproché que dans la mesure où cette dernière présente, elle aussi, une grande étoile à cinq branches autour de laquelle s'articule la composition du réseau. La chronologie relative entre les deux roses reste à déterminer.

${ }^{19}$ Le tombeau de Pierre de Montreuil, aujourd'hui détruit, se trouvait dans la Chapelle de la Vierge de l'abbaye Saint-Germain-des-Prés de Paris. L'architecte y avait été représenté avec sa règle et son compas.

${ }^{20}$ Hugues Libergier, architecte de Saint-Nicaise de Reims depuis le début de construction jusqu'à sa mort en 1263 avait été enterré dans l'abbatiale ; après la destruction de Saint-Nicaise, sa dalle fut transférée vers 1800 dans la cathédrale de Reims.
} 
L'anecdote rapportée par dom Pommeraye étant sans doute fausse, qu'en est-il de la date de 1439 qu'indique le même auteur pour la réalisation des deux roses de SaintOuen? Bien que le savant bénédictin assure avoir puisé ses informations dans «quelques anciens manuscrits de l'Abbaye et de la bibliothèque de $M$. Bigot» - un célèbre bibliophile rouennais qui ouvrait sa collection de livres à la curiosité des savants $^{21}$-, ce millésime apparaît in fine comme le résultat d'une simple déduction : Alexandre ayant été « exécuté » au tout début de l'année 1440 (5 janvier 1441 n.s.), le «meurtre » devait avoir lieu peu de temps auparavant, et ce "meurtre » était à son tour précédé de peu par la réalisation des deux roses qui constituaient le « mobile du crime ».

Pour dater la rose méridionale de Saint-Ouen de Rouen, on ne peut donc pas se fonder sur la date de 1439 ni sur aucun autre document, ce qui conduit à revenir sur la fourchette chronologique fournie par la maîtrise d'œuvre d'Alexandre de Berneval, en l'occurrence la période comprise entre 1422 environ et le début de l'année 1441. Toutefois, Peter Kurmann a récemment mis en question cette datation, estimant qu'Alexandre a simplement réalisé à grande échelle le dessin de remplages inventé peutêtre par l'un de ses prédécesseurs, les maîtres d'œuvre Jean I ou Jean II de Bayeux ${ }^{22}$. On objectera que sur la dalle funéraire, le défunt ne tient pas un "modèle », comme l'a supposé cet auteur, mais bien un dessin précis, "technique», correspondant à un tiers de la rose. Avant tout, et à l'instar de la fameuse illustration de la Bible moralisée de Vienne montrant Dieu le Père créant le monde à l'aide d'un compas ${ }^{23}$, la représentation figurée insiste sur l'acte de création : l'architecte tient de la main droite le petit compas, les branches étant posées directement sur le dessin géométrique de la rose. De manière on ne peut plus claire, Alexandre de Berneval est donc désigné comme le concepteur du dessin géométrique de la rose, qu'il semble justement occupé à élaborer.

Par ailleurs, l'agencement formel de la rose et de ses remplages pourrait certes avoir été conçu dès les alentours de 1400, comme le propose Peter Kurmann, mais il est tout aussi compatible avec une datation dans les années 1420 ou 1430. Ainsi, le somptueux écran d'arcades aveugles de la façade ouest de la cathédrale de Rouen, réalisée durant de longues décennies entre 1370 et 1422 environ, montre justement dans ses parties les plus tardives, érigées par Jeanson Salvart à partir de 1407, un vocabulaire protoflamboyant tout à fait comparable à celui de la rose sud de Saint-Ouen de Rouen (fig. 6). A l'instar de cette dernière, les compositions de Jeanson Salvart se composent exclusivement de trèfles et de quadrilobes cadrés, regroupés au sein de mouchettes formant des grands cadres curvilignes. Nous retrouvons ce même vocabulaire dans les fenêtres hautes du chevet de la cathédrale de Rouen, reconstruites par Salvart entre 1429 et $1433^{24}$. Ce n'est qu'à la fin des années 1430 qu'une rupture majeure dans

\footnotetext{
${ }^{21} \mathrm{Cf}$. Louis Jacob DE SAINT-CHARLES, Traicté des plus belles bibliothèques publiques et particulières, qui ont esté \& qui sont à présent dans le monde, Paris, Chez Rolet le Duc, 1644, p. 681.

${ }^{22}$ Peter KURMANN, « Rouen, abbatiale Saint-Ouen. Une plaque tournante du gothique européen autour de 1400 : la façade du bras sud et son portail », Congrès archéologique de France, $161^{e}$ session, 2003, Rouen et Pays de Caux, Paris, Société française d'archéologie,2005, p. 239-247, en part. p. 245.

${ }^{23}$ Vienne, Österreichische Nationalbibliothek, Codex Vindobenesis 2554, fol. $1 \mathrm{v}^{\circ}$.

${ }^{24}$ Pour ces travaux de Jeanson Salvart, voir en dernier lieu Markus SCHLICHT, «Architecte, commande, style, modèle. Quelques remarques sur la réfection des fenêtres hautes du chevet de la cathédrale de Rouen (1429-
} 
l'architecture religieuse rouennaise (et française) se produit : en 1436, en effet, le maître d'œuvre Pierre Robin fut payé pour les plans de l'église paroissiale Saint-Maclou qui est la première à présenter (exclusivement) de véritables réseaux flamboyants.

Rien ne s'oppose donc à attribuer à Alexandre de Berneval l'invention du dessin de remplages de la rose sud de Saint-Ouen de Rouen. Si la datation de cette dernière dans la période comprise entre 1422 environ et 1441 permet de confirmer dans une certaine mesure la réalisation de la rose occidentale de la cathédrale de Tours au cours du deuxième quart du XVe siècle et, de ce fait, son attribution à Jean de Dammartin, rien ne nous permet de trancher la question de l'antériorité de l'une des deux œuvres.

\section{Les autres œuvres attestées des deux architectes : Le bras nord du transept de la cathédrale du Mans et le Pas de l'ange de la Trinité de Fécamp}

L'examen d'autres œuvres émanant de ces deux architectes nous fournit-il des indices supplémentaires pour répondre à cette question? Avant d'avoir été appelé à la maîtrise d'œuvre de la cathédrale de Tours vers 1426/31, Jean de Dammartin - nous l'avons mentionné ci-dessus - exerça cette même fonction à la cathédrale Saint-Julien du Mans. Il y avait été engagé en janvier 1421, après le décès de son prédécesseur Nicolas de l'Écluse en 1420, pour mener à bien la construction du bras nord du transept. Celui-ci, mis en chantier en $1403^{25}$, semble menacer ruine en 141926. Même si les parties du bras nord revenant respectivement à Nicolas de l'Écluse et Jean de Dammartin n'ont pas encore été déterminées ${ }^{27}$, il paraît évident que ce dernier, intervenant en fin de chantier, a dû réaliser les parties supérieures de l'œuvre. Jean a dû superviser plus particulièrement la construction de la charpente du bras nord, pour la confection de laquelle le chapitre avait acheté 200 chênes en $1423^{28}$. Le pignon du bras nord, clôturant cette charpente, a donc dû être érigé sous sa direction, voire d'après ses plans.

Ce pignon, certes assez parcimonieusement orné, ne présente pas de remplages flamboyants ou proto-flamboyants (fig. 7). Sa surface triangulaire est ornée d'un grand arc brisé dont l'intrados montre, à l'instar d'un décor à festons, une suite de demi-cercles

1433) », Ars auro gemmisque prior. Mélanges en hommage à Jean-Pierre Caillet, dir. C. BLONDEAU et al., Zagreb-Motovun, International Research Center for Late Antiquity and the Middle Ages Motovun, University of Zagreb, 2013, p. 431-437.

${ }^{25}$ Gustave ESNAULT, « Le transept septentrional de la cathédrale du Mans, architectes et bienfaiteurs (13931430) », Bulletin monumental, 1879, p. 63-79, en part. p. 65-66. Francis SALET, « La cathédrale du Mans », Congrès archéologique de France, CXIX session, 1961 Maine, Paris, Société française d'archéologie, 1961, p. 18-58, en part. p. 56.

${ }^{26}$ La plupart des historiens ont rapporté le passage mentionnant l'église en grand péril (« ...in reparationibus et eminenti pericolo... dicte nostre ecclesie... ») figurant dans les registres capitulaires, au bras nord du transept (ainsi, le premier, G. ESNAULT (art. cit. note 23), p. 68-69 ; en dernier lieu T. RAPIN, « Les Dampmartin... » (art. cit. n. 2), p. 267). Michel Bouttier pense, en revanche, que le texte se réfère aux voûtes de la nef romane fissurées qui auraient failli s'effondrer et qu'on aurait alors stabilisées par la construction des arcs-boutants actuels, cf. M. BoutTiER, La cathédrale du Mans, Le Mans, La Reinette, 2000, p. 104.

${ }^{27}$ M. BoutTIER (op. cit. n. 26), p. 104 attribue à Nicolas de l'Écluse le mur occidental du bras nord « jusqu'au faîte » ainsi que la grande rose de la façade. Les dessins des remplages des fenêtres hautes des murs oriental et occidental sont toutefois si similaires qu'ils ne fournissent pas d'indication permettant d'affirmer qu'ils ont été conçus par deux maîtres d'œuvre différents.

${ }^{28}$ G. Esnault (art. cit. note 23), p. 74 ; F. SALET, « Cathédrale du Mans... » (art. cit. n. 23), p. 58. 
redentés. En son centre, l'architecte a aménagé une baie à cintre brisée, meublée de deux lancettes à têtes redentées et d'un quadrilobe cadré. Quant au garde-corps courant au pied du pignon, il est constitué d'un enchaînement de quadrilobes cadrés. Les pinacles couronnant les tourelles d'angle de la façade ne montrent pas davantage de formes proto-flamboyantes qui pourraient être considérées comme annonciatrices du dessin de la rose de Tours.

Jean de Dammartin a pu aussi réaliser l'intégralité ou du moins partie des fenêtres hautes du bras nord du Mans (fig. 7, en encadré). Ces très grandes baies, composées de huit lancettes, sont dotées de remplages à polylobes multiples formant des compositions complexes. Par rapport au pignon, le maître d'œuvre utilisa ici un répertoire "plus moderne » de formes géométriques faisant largement appel aux éléments protoflamboyants, tels que les mouchettes en rotation (deux, trois ou quatre) inscrites dans un cercle, les quadrilobes à un ou deux lobes effilés en pointe, ou encore les trèfles et quatre-feuilles irréguliers meublant les pointes des tympans secondaires à cintre brisé. En revanche, la disposition d'ensemble des réseaux de ces grandes baies, structurée essentiellement à l'aide de grands cadres circulaires et d'arcs brisés, entrecroisés ou non, reste largement tributaire des conventions du gothique rayonnant. La ligne sinueuse à courbe et contrecourbe apparaît timidement dans certains tympans, mais elle n'anime jamais les lignes de force de la composition, pas plus que les cadres englobant plusieurs polylobes.

Enfin, la grande baie à rose intégrée ornant la façade du bras nord du transept manceau présente des caractères formels fort similaires à celui des fenêtres hautes ${ }^{29}$. Le répertoire des dessins géométriques utilisés comporte les mêmes mouchettes en rotation inscrites, les mêmes quadrilobes dont les lobes supérieurs et inférieurs s'étirent en pointe, et les mêmes trèfles libres et fortement déformés logés dans les écoinçons (fig. 7). Si ce répertoire proto-flamboyant apparaît assez moderne, la composition d'ensemble du réseau reste attachée aux principes rayonnants: grand oculus central regroupant quatre trèfles cadrés; douze grands pétales droits à corolle simple subdivisés en deux rayons surmontés de deux trèfles cadrés et d'une courte mouchette droite ; écoinçons systématiquement ornés d'un trèfle libre.

Les spécificités formelles du réseau de la rose septentrionale du Mans diffèrent donc foncièrement de celles des roses de Tours et de Rouen qui ne font pas appel ni aux mouchettes en rotation ni aux quadrilobes à pointes effilées. Seule la rose de Tours présente, près du cercle périphérique, des trèfles libres fortement déformés, tels qu'on les trouve au Mans. Alors que l'auteur de la rose du Mans regroupe plusieurs polylobes dans l'oculus central et dans les pointes des pétales, le concepteur du dessin des remplages de Tours et de Rouen renonce à ces accumulations de polylobes. Avant tout, l'inventeur de l'agencement des roses tourangelle et rouennaise confère aux lignes principales de sa composition un dessin courbe ou sinueux - les grands pétales adoptent approximativement la forme d'une mouchette -, tandis que l'auteur de la rose mancelle

\footnotetext{
${ }^{29}$ Selon T. RAPIN, «Les Dampmartin...» (art. cit. n. 2), p. 268-269, rien ne permet de l'attribuer à Jean de Dammartin plutôt qu'à Nicolas de l'Ecluse.
} 
utilise exclusivement des pétales droits. Compte tenu de ces divergences, il ne semble guère possible d'établir un lien entre le réseau de la baie du Mans et ceux des roses de Tours ou de Rouen.

En l'absence d'autres œuvres attestées (et conservées) de Jean de Dammartin, il convient de se tourner à présent vers l'examen des monuments exécutés par Alexandre de Berneval. Bien que des sources évoquent à plusieurs reprises des commandes artistiques confiées à cet architecte ${ }^{30}$, on ne peut lui attribuer qu'une œuvre en dehors de la rose méridionale de Saint-Ouen : le Pas de l'ange, un tabernacle-reliquaire qu'il réalisa en 1420/21 pour l'abbaye de la Trinité à Fécamp ${ }^{31}$. Selon la légende, un angepèlerin serait miraculeusement apparu aux personnes réunies pour la dédicace de l'abbatiale de Fécamp, reconstruite vers 940, et leur aurait enjoint de la consacrer à la Sainte Trinité, avant de disparaître dans les cieux, laissant comme témoignage de son apparition l'empreinte de son pas dans une pierre dure.

Le reliquaire de Fécamp prend la forme d'un baldaquin de plan carré à décor architecturé, dans lequel on disposa la pierre avec l'empreinte de l'ange ainsi qu'une représentation sculptée commémorant le miracle : entouré du duc Guillaume LongueEpée et des évêques normands assistant à la cérémonie de la consécration, le pèlerin s'apprête à franchir l'entrée de l'église pour déposer sur l'autel à dédicacer un couteau portant l'inscription "In nomine sanctae et individuae Trinitatis». Alors que les autres formes architecturales du baldaquin montrent un répertoire de polylobes rayonnants conventionnel, composé de quadrilobes cadrés et de rayons, le haut-relief illustrant l'événement présente une façade d'église intégrant quelques formes protoflamboyantes : arc d'entrée en anse de panier, pignon à rampants incurvés, petit trèfle irrégulier meublant la pointe du pignon, et avant tout la rose principale animée de mouchettes courbes disposées en spirale (fig. 8). Si le petit monument illustre la familiarité d'Alexandre de Berneval avec le vocabulaire proto-flamboyant dès les alentours de 1420, on ne peut guère le considérer comme un véritable antécédent pour la rose du bras sud du transept de Saint-Ouen de Rouen : contrairement à cette dernière, la petite rose de Fécamp, purement décorative, n'est soumise ni à des contraintes d'ordre statique, ni aux problèmes pratiques générés par l'assemblage d'une grande quantité de blocs de pierre.

\footnotetext{
${ }^{30}$ Dès 1413, et à la demande de l'abbé de Fécamp Estaud d'Estouteville, Alexandre de Berneval entreprit un voyage en Angleterre afin d'acheter de l'albâtre pour un monument malheureusement non précisé, cf. notamment Charles DE BEAUREPAIRE, « Notes sur les architectes de Rouen », Bulletin de la Société des Amis des monuments rouennais, 2, 1902, p. 67-93, en part. p. 85-87. Dès 1417, Alexandre porte le titre de maître d'œuvre du baillage de Rouen, et il semble avoir surveillé les travaux du palais royal (pour Henri VI) de Rouen; de ce dernier ne subsistent que des vues anciennes ; cf. Pierre-Yves LE POGAM, «Un chantier exemplaire : le palais royal de Rouen », Bulletin archéologique du comité des travaux historiques et scientifiques, nouv. série, 23/24, années 1987-1988 (Antiquités nationales), paru en 1991, p. 213-247.

${ }^{31}$ Pour ce monument, voir en dernier lieu Jean-Marie GuILlOUËT, « Une sculpture du XV siècle et son contrat. Le "Pas de l'ange" à la Trinité de Fécamp », Bibliothèque de l’Ecole des chartes, 162, 2004, p. 133-161.
} 


\section{Le réseau des roses de Tours et de Rouen : à la manière de Jean de Dammartin ou d'Alexandre de Berneval ?}

L'examen des œuvres attestées des deux architectes - il est vrai de manière moins assurée en ce qui concerne Jean de Dammartin - ne permet certes pas de trancher de manière définitive la question de l'attribution du dessin des remplages qu'arborent aussi bien la rose de Tours que celle de Rouen. Il permet néanmoins de dresser un premier constat : alors que ce dessin paraît incompatible avec ceux que l'on peut attribuer à Jean de Dammartin au Mans, il paraît davantage compatible avec certains éléments du tabernacle-reliquaire d'Alexandre de Berneval. Afin de confirmer ou d'infirmer cette hypothèse, il convient de confronter à présent le dessin des roses avec d'autres compositions de remplages des façades tourangelle et rouennaise attribuables à l'un ou l'autre des deux architectes.

Les remplages plaqués qui surmontent les portails de la façade occidentale de la cathédrale de Tours ont été attribués à plusieurs reprises à Jean de Dammartin ${ }^{32}$. Le niveau inférieur de ces remplages, dessinant un "faux triforium », a déjà été évoqué cidessus (fig. 3). Leur répertoire de polylobes, composé de quadrilobes au lobe inférieur sinusoïdal et de mouchettes convergentes, ne se retrouve pas au sein de la grande rose occidentale. Si la rose contient, à l'instar du "faux triforium», des trèfles libres fortement déformés afin de meubler les écoinçons (proche du cadre formant un grand losange curviligne), elle ne présente en revanche pas les quatre-feuilles aux lobes asymétriques et fortement étirés qui décorent le «triforium factice » (elles sont situées en dessous des deux mouchettes convergentes).

Les remplages plaqués qui surmontent ce «faux triforium » forment, au nord comme au sud, une baie aveugle à quatre lancettes surmontées d'une rose multipartite. Du côté nord, cette rose aveugle présente, rayonnant autour d'un oculus quadrilobé central, seize pétales droits comportant chacun un rayon centrifuge et un quadrilobe cadré (fig. 3). Si le répertoire des quadrilobes, en l'occurrence les quadrilobes cadrés disposés près du cercle périphérique et les rayons droits, se retrouvent aussi dans la rose de la grande baie centrale, les dessins des réseaux se distinguent fondamentalement par la manière dont ces éléments sont disposés : à l'ordonnance strictement rayonnante et rigide de la baie latérale, soulignée par le cadre droit des pétales formant autant de rayons de cercle, s'opposent les courbes élégantes et souples des pétales de la grande rose du centre. La courbure des pétales engendre à son tour l'inflexion de leurs composantes (rayons) ou introduit des variations dans leur orientation (quadrilobes).

Formant pendant à la baie aveugle à rose intégrée de la tour nord, les remplages plaqués du registre correspondant de la tour sud présentent, eux aussi, quatre lancettes surmontées d'une rose multipartite inscrites dans un cadre rectangulaire. De nouveau, le maître d'œuvre souligna la disposition strictement rayonnante des remplages en subdivisant l'aire circulaire de la rose par quatre lignes droites formant huit rayons de

\footnotetext{
${ }^{32}$ T. RAPIN et J. NoBlet (art. cit. n. 2), p. 71-72 ; T. RAPIN, « La cathédrale de Tours... ( (art. cit. n. 2), p. 306 et fig. 6 p. 305. C. ANDRAULT (op. cit. n. 2), p. 244, suit la chronologie proposée par Thomas Rapin et ainsi, de manière implicite, l'attribution des remplages à Jean de Dammartin.
} 
cercle ; il accentua même cette partition du cercle en prolongeant les rayons à travers l'oculus central, dont l'agencement rappelle ainsi une roue à huit rayons. Autour de l'oculus rayonnent huit petits pétales à tête redentée puis, au-dessus, deux mouchettes en rotation inscrites dans un cercle. Aucun de ces éléments ne se retrouve dans le réseau de la grande rose occidentale, pas plus que les quadrilobes libres fortement déformés qui meublent les écoinçons supérieurs de la composition.

Il me semble inutile de poursuivre cet examen dans les parties supérieures de la façade de Tours. Outre le fait que leur attribution à Jean de Dammartin paraît plus fragile, ces remplages ne présentent pas davantage de parallèles formels par rapport à la grande rose de la baie centrale que ceux déjà évoqués. Au bout de ces considérations, le réseau de cette dernière apparaît presque comme un corps étranger au sein du tissu des remplages aveugles couvrant le frontispice tourangeau.

Le dessin de la grande rose est-il davantage conforme au style personnel d'Alexandre de Berneval ? Faute d'une quantité plus importante d'œuvres attestées, ce style personnel reste pour l'heure largement insaisissable. Le transept de l'abbatiale de Saint-Ouen, dont il dirigea les travaux de construction durant une vingtaine d'années, présente certes bien des éléments proto-flamboyants, mais ce vocabulaire fut employé par plusieurs maîtres d'œuvre rouennais, dont notamment les prédécesseurs d'Alexandre, Jean I et Jean II de Bayeux, et probablement aussi son fils et successeur Colin de Berneval.

Mais peut-être n'est-il pas nécessaire d'isoler les créations personnelles d'Alexandre au sein de toute la production architecturale rouennaise de la période proto-flamboyante justement à cause du caractère remarquablement homogène de celle-ci.

Que l'on examine le porche précédant la façade du bras sud du transept de Saint-Ouen (vers et après 1400), la rose aveugle en spirale dans l'annexe précédant le bras nord de ce même transept, voire même l'écran d'arcades ornant la façade occidentale de la cathédrale voisine (entre 1370 et 1422 environ, fig. 6), ou les fenêtres hautes du chevet du même édifice (1429 à 1433), on trouve toujours le même répertoire restreint de polylobes et la même façon de les disposer : il s'agit essentiellement de trèfles et de quadrilobes cadrés, parfois complétés par des rayons courbes; ces éléments classiques du vocabulaire rayonnant sont enchâssés et regroupés au sein de cadres «flamboyants » aux contours courbes ou sinusoïdaux. Contrairement aux réseaux du Mans ou de la façade occidentale de Tours, ceux de la capitale normande ne font jamais appel aux mouchettes en rotation inscrites dans des cercles ni aux trèfles ou quadrilobes libres asymétriques et fortement déformés; en outre, les quadrilobes à lobes effilés n'y apparaissent qu'exceptionnellement, au centre de la rose du bras nord du transept de Saint-Ouen.

Le répertoire de polylobes - trèfles et quadrilobes cadrés - ainsi que la manière de les regrouper à l'aide de cadres sinusoïdaux, qui caractérisent le réseau des roses de Rouen et de Tours, correspondent donc parfaitement aux conventions formelles observées par les maîtres d'œuvre rouennais de la fin du XIVe siècle et du premier tiers du siècle suivant. Il semble alors judicieux d'attribuer la paternité du dessin des remplages au 
rouennais Alexandre de Berneval. Il faut en conclure que la rose de Saint-Ouen précède dans le temps celle de Saint-Gatien de Tours, et que Jean de Dammartin a copié ce dessin tout en le redessinant pour l'adapter au cadre losangé. Les modifications qu'il y apporta furent mineures, comme le remplacement des trèfles cadrés qui meublent les écoinçons entre la pointe des pétales rouennais par des trèfles libres et asymétriques, tels qu'on les trouve aussi dans les baies de Saint-Julien du Mans et dans les réseaux aveugles du frontispice de Tours.

Ces considérations, certes non exemptes d'incertitudes, peuvent jeter une lumière plutôt insolite sur les modalités d'un transfert de motif dans le domaine de l'architecture. Contrairement au mode de diffusion des motifs architecturaux le plus souvent constaté qui va de l'établissement religieux hiérarchiquement supérieur vers l'église occupant un rang inférieur ${ }^{33}$, le dessin des remplages s'est transmis ici en sens inverse, même si les deux établissements concernés n'entretenaient pas de rapport hiérarchique direct: élaboré pour une abbatiale bénédictine, il fut ensuite imité dans une cathédrale métropolitaine. Il peut paraître tout aussi surprenant qu'un architecte méconnu, Alexandre de Berneval, soit l'inventeur d'une grande composition de remplages qu'un architecte plus célèbre, Jean de Dammartin, s'est ensuite contenté de copier - à moins que la plus grande renommée de ce dernier, largement liée à celle de son oncle Guy et de son père Drouet de Dammartin, ne soit qu'une appréciation erronée de l'historiographie actuelle.

Quoi qu'il en soit, la réalisation d'une «copie » - ou la «citation » - d'un modèle ne possédait pas au Moyen Âge la connotation péjorative dont elle est investie de nos jours. La référence à un modèle donné était un procédé habituel et même incontournable par lequel le commanditaire indiquait au maître d'œuvre l'aspect qu'il convenait de donner à la future construction ${ }^{34}$. Il est donc fort probable que l'imitation de la rose rouennaise à la cathédrale de Tours corresponde à une commande expresse en ce sens de son clergé. Celui-ci était sans doute parfaitement conscient du fait que le réseau de la rose d'Alexandre était non seulement d'une grande modernité, mais qu'il introduisait aussi des nouveaux principes esthétiques : sous les mains de l'architecte, le matériau pierre, dur par essence, semble ramollir et devenir malléable ; libérée de sa rigidité habituelle, elle devient souple, se courbe et ondule désormais. Matière inerte, la pierre semble même dans une certaine mesure s'animer pour former, à l'instar d'un végétal, des bouquets de tiges fleuries. Bien qu'en transfigurant ainsi le matériau de construction, Alexandre de Berneval ne visait toutefois pas l'illusionnisme : le spectateur s'émerveille

\footnotetext{
${ }^{33}$ Cf. par exemple les remarques synthétiques sur ce sujet par Dany SANDRON, « Des cathédrales romanes aux cathédrales gothiques », dans 20 siècles en cathédrales, Paris, Monum, Éditions du patrimoine, 2001, p. 157168 , en part. p. $165-167$.

${ }^{34}$ En témoigne cet exemple parmi bien d'autres : en 1402, les trésoriers de Saint-Patrice de Rouen chargèrent le maître maçon Guillaume Doré de moderniser le chevet de leur église par la construction d'une « fourme de pierre de taille de Vernon de telle hauteur et ainsi feuillie et ordonnée comme celle de Saint-Laurent de Rouen, excepté qu'en la fourme de S. Patrice il devoit y avoir une voussure »; cité d'après Charles DE BEAUREPAIRE, « Documents relatifs à la construction de l'église Saint-Ouen de Rouen », dans Notes historiques et archéologiques concernant le département de la Seine-Inférieure et spécialement la ville de Rouen, dir. ID., Rouen, É. Cagniard, 1883, p. 24-35, en part. p. 28-29
} 
d'autant plus de cette rose qu'il sait pertinemment qu'elle est faite de pierre. L'architecte rouennais n'avait pas non plus l'intention d'imiter une plante véritable : sa composition est une construction géométrique, générée exclusivement à l'aide d'un compas et d'une équerre, que révèlent clairement les multiples axes de symétrie et les courbes régulières qui animent le dessin des remplages. Alexandre de Berneval fut l'un des premiers à avoir abandonné la mise en page purement rayonnante d'une rose monumentale, ouvrant la voie à l'élaboration d'une multitude de nouvelles organisations de ces composantes phare des grands édifices gothiques. On ne s'étonnera donc pas que les moines de SaintOuen lui aient fait l'honneur d'un somptueux monument funéraire. 